\title{
Translation, cross-cultural adaptation, and validation of a semi-structured interview Physical Activity Recall Assessment for People with Spinal Cord Injury in persons with paraplegia
}

\author{
Jarmila Štěpánová*, Lukáš Jakubec, Kateřina Neumannová, Radka Čiháková, Michaela \\ Lehnertová, and Martin Kudláček
}

Faculty of Physical Culture, Palacký University Olomouc, Olomouc, Czech Republic

Copyright: (C) 2020 J. Štěpánová et al. This is an open access article licensed under the Creative Commons Attribution License (https://creativecommons.org/licenses/by/4.0/).

\begin{abstract}
Background: In the Czech Republic a standardized measuring method has not been applied yet in order to monitor the amount and intensity of physical activity of persons with spinal cord injury. Objective: The purpose of this study was to translate, adapt and validate administration and scoring manual of the semi-structured interview Physical Activity Recall Assessment for People with Spinal Cord Injury (PARA-SCI), focused on the subjective perception of the amount of physical activity of persons with paraplegia. Methods: To determine the criterion validity of the research tool, we compared data from 53 people with paraplegia collected through PARA-SCI interviews and data from the ActiGraph GT3X+ accelerometer, which respondents wore on their non-dominant wrist. We evaluated the test-retest reliability among 39 subjects with paraplegia, which consists in administering the questionnaire on two occasions separated by a time interval one week. Results: In the validity study, the correlation between data for overall physical activity over three days form accelerometers and PARA-SCI interviews showed the strong level of correlation strength $(r=.58, p<.001)$. The intraclass correlation between data for overall physical activity over three days from two PARA-SCI interviews showed a moderate level of test-retest reliability $(r=.70, p<.001)$. The intraclass correlations ranged from .38 to .80 for the various PARA-SCI activity categories and intensities. Conclusions: A Czech version of the semi-structured interview PARA-SCI was standardised and can be used by physiotherapists, nutrition therapists and sports educators from the field of adapted physical activities in the Czech Republic.
\end{abstract}

Keywords: disability, measurement, standardization, wheelchair users, physical activity

\section{Introduction}

Czech epidemiological data from spinal rehabilitation departments in hospitals between the years 2006-2015 (Kriz, Kulakovska, Davidova, Silova, \& Kobesova, 2017) indicated more than 250 new cases of spinal cord injury per year, male to female ratio of 4:1. Mechanical damage to the spinal cord was located in about $40 \%$ in the cervical part (tetraplegia), in $40 \%$ in the thoracic part of the spinal cord and in $20 \%$ in the lumbar part of the spinal cord (paraplegia). Paraplegia is commonly divided into high (Th1-Th6) and low (Th7-L2; Harvey, 2007; Zäch \& Koch, 2006).

\footnotetext{
* Address for correspondence: Jarmila Štěpánová, Department of Adapted Physical Activities, Faculty of Physical Culture, Palacký University Olomouc, třída Míru 117, 77111 Olomouc, Czech Republic. E-mail: jarmila.stepanova@upol.cz
}

It is important to note, that for most people with spinal cord injury (SCI), physical deconditioning was a common consequence of the injury (Buchholz, McGillivray, \& Pencharz, 2003; Latimer, Martin Ginis, Craven, \& Hicks, 2006), and led to the increased risk of developing obesity and other chronic secondary health complications (Garshick et al., 2005; Tawashy, Eng, Lin, Tang, \& Hung, 2009). It also brought about further handicap by contributing to decreased mobility, physical function, independence and self-care of a person with spinal cord injury (SCI; Hetz, Latimer, Buchholz, \& Martin Ginis, 2009; Pokuta, Slavíková, \& Honzátková, 2014).

On the other hand, regular physical activity (PA) decreased depressive symptoms, levels of chronic pain and fatigue and risk of chronic secondary health complications such as cardiovascular diseases, Type II diabetes and osteoporosis (Ginis et al., 2003; Hetz, 
Latimer, Buchholz, \& Martin Ginis, 2009; Hicks et al., 2003; Tawashy et al., 2009). PA also positively improved self-sufficiency in activities of daily living (Hetz, Latimer, \& Martin Ginis, 2009), community participation (Carpenter, Forwell, Jongbloed, \& Backman, 2007) and thereby positively influenced the level of health-related quality of life of persons with SCI (Anneken, Hanssen-Doose, Hirschfeld, Scheuer, \& Thietje, 2010).

In the Czech Republic, an epidemiologic study had not been carried out yet in order to find out the health status and the degree of an active lifestyle of persons with SCI during the chronic phase of their disability. One of the reasons was the lack of a suitable targeted and standardized examination method. Štěpánová, Kudláček, and Bednaříková (2016) performed a systematic review of international studies from the period 2004-2014 with the aim of finding an appropriate self-reporting physical activity measure for persons with SCI. As a result, the administration and scoring manual of semi-structured interview Physical Activity Recall Assessment for People with Spinal Cord Injury (PARA-SCI; Martin Ginis \& Latimer, 2008) was selected and purchased. The purpose of this study was to create and validate a Czech version of the semistructured interview PARA-SCI, focused on subjective perception of the amount of physical activity of persons with paraplegia.

\section{Methods}

\section{Cross-cultural adaptation}

Cross-cultural adaptation had been performed according to the Guidelines for the Process of Cross-Cultural Adaptation of Self-Report Measures (Beaton, Bombardier, Guillemin, \& Ferraz, 2000). Two initial translations, synthesis of the translations and two back translations were carried out. Consequently, the expert committee was established. The committee composed of three academic staff (one associate professor, two lecturers) and four translators (forward and back translators). All the translations were sent to the main author of the original version for comment, too. The preliminary version of the semi-structured interview was completed.

The pilot testing of the PARA-SCI preliminary version consisted of 6 interviews. The participants were both adults with spinal lesion and clients of the Paraple Center, o.p.s. in Prague. Based on the pilot testing results the Adapted version of semi-structured interview PARA-SCI was finished.
Assessing content validity, criterion validity and reliability of the Adapted Version PARA-SCI (PARA-SCI.CZ)

Content validity was estimated according to Lawshe's (1975) methodology for 10 selected items from the second and third part of the PARA-SCI manual. Three academic staff and three experts in physiotherapy and adapted physical activities (practitioners) assigned a corresponding letter to each item, which evaluated the content validity. If necessary, it was accompanied by a verbal commentary.

Criterion validity reflected a correlation between the Czech version of PARA-SCI and an accelerometer Actigraph GT3X+ measure of total amount (minutes) of habitual PA of people with SCI for three days. The most fundamental way of assessing reliability was testretest reliability, which consists in administering the questionnaire on two occasions separated by a time interval sufficiently short that the general patterns of PA should not change (Streiner \& Norman, 2008). We used the data from two semi-structured interviews PARA-SCI.CZ by a time interval one week according to Martin Ginis, Latimer, Hicks, and Craven (2005).

More specifically, testing of the criterion validity and reliability of the PARA-SCI.CZ consisted of three steps. First, the actual PA of the wheelchair users was monitored over a three-day (two days during the workweek and one day during the weekend) period by using accelerometers Actigraph GT3X+. Second, the next day after finishing the accelerometer measurement the participants had their first telephone semi-structured interview PARA-SCI.CZ asking about the three previous days, i. e. the days and that the accelerometer was worn. Third, a week later second telephone interview PARA-SCI.CZ was conducted with the participants, with a condition that the participants were not allowed to change their physical habits due to an illness, vacation, national holidays or a stay in a rehabilitation facility. The measurements took place in home conditions. Participants received a table containing validated SCIspecific definitions of four PA intensity levels together with an accelerometer in the package. The research was approved by the Ethics Committee of the Faculty of Physical Culture, Palacký University Olomouc (reference number 4/2016).

\section{Participants}

A total of 53 persons with a mean age 41.8 years $(S D$ 9.65; 10 females, 43 males) with a permanent acquired physical disability caused by partial or complete spinal cord injury with lesion localisation between the cord segments Th1-L2 (paraplegia) participated in the data collection. The spinal lesion had occurred more than two years ago. Their health and therefore also the functional status were stabilized and the rehabilitation 
treatment at the subacute stage had terminated. All participants used a mechanical wheelchair as their primary means of locomotion. All participants lived in the Czech Republic, the Czech language was their mother tongue and they were without cognitive impairment. All participants signed a voluntary informed consent before the start of the study.

\section{Activity protocol}

The adapted version PARA-SCI.CZ measures activity actually performed over a three day time period and was designed to capture two categories of PA - leisure-time physical activity (LTPA) and activities of daily living (ADL) in 4 PA intensity levels: nothing at all (no physical effort), mild (very light physical effort), moderate (some physical effort), and heavy (maximum physical effort). The PARA-SCI.CZ data was evaluated according to the translated manual (Martin Ginis \& Latimer, 2008) and scored by calculating the mean number of minutes per day during three days of ADL, LTPA and total PA (sum of ADL and LTPA) performed at mild, moderate, and heavy intensities.

\section{Accelerometer-based physical activity monitoring}

ActiGraph GT3X+ (ActiGraph, Pensacola, FL, USA) is small $(4.6 \times 3.3 \times 1.5 \mathrm{~cm})$ and low weight $(19 \mathrm{~g})$ device. It is suitable for wearing on a wrist for wheelchair users. For the purpose of the research fastening straps were made (Figure 1). The accelerometer was fixed on participants' wrist, more precisely on the dorsal side of their non-dominant wrist according to the example (Filáková, Štěpánová, \& Jakubec, 2019; García-Massó et al., 2013). PA was being recorded over a period of three consecutive calendar days (two

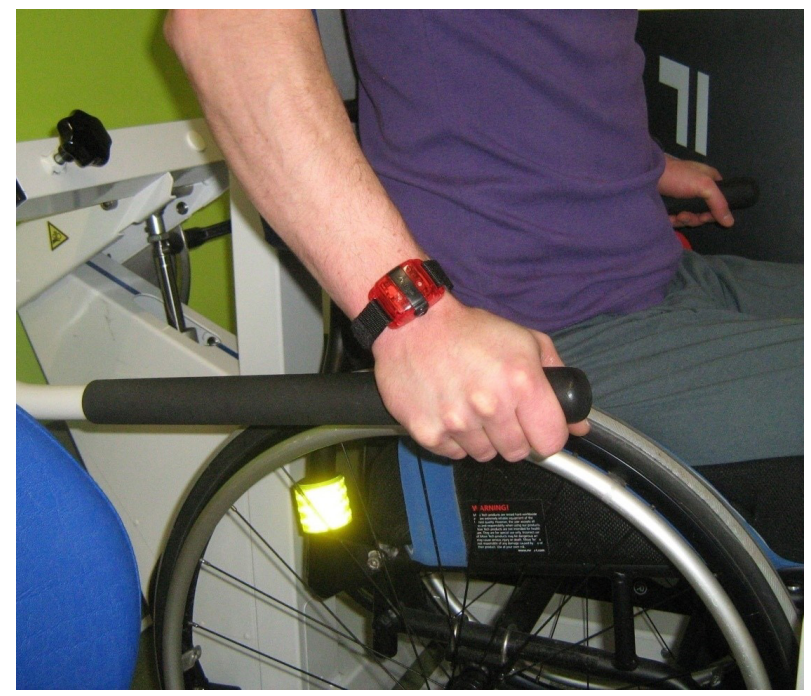

Figure 1. ActiGraph GT3X+ fixed on the dorsal side of the non-dominant wrist. days during the workweek and one day during the weekend) in home conditions at a $30 \mathrm{~Hz}$ frequency. The participants put the device on the wrist after waking up, before performing the daily routine, and took it off in the evening just after lying down in bed. Due to limited water-resistance of the accelerometer and a slowly drying material of the fastening strap, the participants were allowed to put the device away for the time necessary to perform personal hygiene (showering, bathing).

The measured accelerometer ActiGraph GT3X+ data was converted into a 30 second intervals. Converted data was subsequently evaluated using the ActiLife program supplied by the accelerometer manufacturer. According to the manufacturer's recommendation, the data indicating very low intensity ( $<100$ counts per minute), was determined as inactivity (ActiGraph, 2016). Vector magnitude cut-off point for moderate-to-vigorous PA (MVPA) was set to 3644 counts per minute according to Learmonth, KinnettHopkins, Rice, Dysterheft, and Motl (2016). Hence the interval 101-3643 counts per minute was considered as low PA.

\section{Statistical analyses \\ Evaluating criterion validity}

Normal distribution of data was verified by ShapiroWilk normality test. Criterion validity of overall PA, low PA and moderate-to-vigorous PA over a three-day period was determined by the Pearson correlation coefficient $(N=53)$. The magnitude of the Pearson correlation coefficient determined three levels of correlation strength by Cohen (Hemphill, 2003). The levels of correlation strength are: small $(.1<r<.3)$, moderate $(.3<r<.5)$ and strong $(r>.5)$. Furthermore, the Pearson correlation coefficient was calculated separately for the group of high spinal lesions Th1-Th6 $(n=25)$ and low spinal lesions Th7-L2 $(n=28)$.

\section{Evaluating test-retest reliability}

Intraclass correlations (one-way random, single measure) for low, moderate, vigorous, and overall activity were calculated for the three categories of activity: ADL, LTPA, and Total PA using two-way random effect models and an absolute agreement definition of reliability $(n=39)$. In compliance with Koo and $\mathrm{Li}$ (2016), the level of reliability was interpreted as follows: values less than .50 are indicative of poor reliability, values between .50 and .75 indicate moderate reliability, values between .75 and .90 indicate good reliability and values greater than .90 indicate excellent reliability. Significance level $\alpha=.05$ was applied for all analyses. 


\section{Results}

\section{Content validity study}

The content validity ratio (CVR) index for the particular items could be found in Table 1. Negative CVR values were not reached in the rating of any item. The item no. 7 reached a satisfactory content validity $(\mathrm{CVR}=0)$. Other items displayed good to very good content validity.

\section{Criterion validity study}

Table 2 shows that the Pearson correlation coefficient for overall PA over 3 days was .57 ( $p<.001)$. In addition, the Pearson correlation coefficient for low PA over 3 days was $.39(p=.01)$ and for MVPA over 3

Table 1

Result of content validity PARA.SCI.CZ

\begin{tabular}{lll}
\hline Item & CVR \\
\hline 1 & PA intensity classification system & 1.00 \\
2 & Types of PA and their recording & 0.33 \\
3 & Data collection design Part 1 & 0.67 \\
4 & Data collection design Part 2 & 0.67 \\
5 & Administration of PARA-SCI & 1.00 \\
6 & General PARA-SCI script & 1.00 \\
7 & General tips for the interviewer & 0.00 \\
8 & Recording PARA-SCI interview data & 1.00 \\
9 & Empty recording sheet PARA-SCI & 1.00 \\
10 & Intensity classification chart & 0.30 \\
\hline
\end{tabular}

Note. $\quad \mathrm{CVR}=$ content validity ratio. days was $.65(p<.001)$. There was a strong level of correlation strength for overall PA over 3 days and for MVPA over 3 days between data from accelerometers and PARA-SCI interviews. Table 3 provides the details of the dependence of the Criterion Validity Test results on the level of spinal cord injury. The number of observations in groups with a high and low level of spinal lesions was almost identical and correlation coefficients of both groups differed only slightly. The correlation coefficient of the first group SCI Th1-Th6 was .56 $(p=.01)$ and the correlation coefficient of the second group SCI Th7-L2 was .58 $(p<.001)$.

\section{Test-retest reliability study}

The second telephone interview was completed by 41 participants. Twelve participants refused to continue to reliability study and get the second phone call. Two participants changed their physical behaviour regime due to illness during the second data collection. Their data was not evaluated. The mean and standard deviation for each type of PA and intensity of PA from the first and second data collection for 39 participants were presented in Table 4. The intraclass correlation coefficient, which was chosen to estimate test-retest reliability for overall PA over a three-day period, was $.70(p<.001)$, which showed a moderate level of reliability. The results of the intraclass correlation coefficients of the selected subgroups of activities presented in Table 4 showed only a poor level of reliability for low PA in ADL, a moderate level of reliability for high and total PA in ADL and low and moderate PA in LTPA, and a good level of reliability for moderate PA in ADL

Table 2

Criterion validity study: Statistical characteristics for total, low and moderate-to-vigorous physical activity over a three-day period $(N=53)$

\begin{tabular}{|c|c|c|c|c|c|}
\hline \multirow[b]{2}{*}{ Activity } & \multicolumn{2}{|c|}{ Shapiro-Wilk test $W$} & \multicolumn{3}{|c|}{ Pearson correlation } \\
\hline & Accelerometer & Interview & $r$ & $95 \% \mathrm{CI}$ & $p$ \\
\hline Total PA & 0.97 & 0.97 & .57 & {$[.42, .80]$} & $<.001$ \\
\hline LPA & 0.96 & 0.96 & .39 & {$[.13, .60]$} & .01 \\
\hline MVPA & 0.96 & 0.98 & .65 & {$[.34, .75]$} & $<.001$ \\
\hline
\end{tabular}

Note. $\mathrm{CI}=$ confidence interval; PA = physical activity; LPA = low intensity PA; MVPA moderate-to-vigorous intensity PA.

Table 3

Criterion validity study: Dependence of the criterion validity test results on level of spinal cord injury

\begin{tabular}{lccccccc}
\hline & & \multicolumn{2}{c}{ Shapiro-Wilk test $W$} & & \multicolumn{3}{c}{ Pearson correlation } \\
\cline { 3 - 4 } \cline { 6 - 8 } Group & $n$ & Accelerometer & Interview & & $r$ & $95 \%$ CI & $p$ \\
\hline SCI Th1-Th6 & 25 & 0.96 & 0.97 & & .56 & {$[.21, .78]$} & .01 \\
SCI Th7-L2 & 28 & 0.96 & 0.94 & & .58 & {$[.26, .78]$} & $<.001$ \\
\hline
\end{tabular}

Note. $\mathrm{CI}=$ confidence interval; SCI Th1-Th6 $=$ high paraplegia; SCI Th7-L2 = low paraplegia. 
Table 4

Test-retest reliability study: Means, standard deviations, intraclass correlation coefficients, and coefficients of variation for scores on the PARA-SCI.CZ

\begin{tabular}{|c|c|c|c|c|c|c|}
\hline \multirow[b]{2}{*}{ Activity } & \multicolumn{2}{|c|}{$M \pm S D$} & \multirow[b]{2}{*}{$p$} & \multicolumn{2}{|c|}{ ICC } & \multirow[b]{2}{*}{$\mathrm{CoV}(\%)$} \\
\hline & Week 1 & Week 2 & & Value & $95 \% \mathrm{CI}$ & \\
\hline \multicolumn{7}{|c|}{ Activities of daily living } \\
\hline Low & $328.69 \pm 89.30$ & $294.45 \pm 97.99$ & .04 & .38 & {$[.09, .62]$} & 49.8 \\
\hline Moderate & $69.99 \pm 75.81$ & $62.02 \pm 68.33$ & .29 & .79 & {$[.64, .88]$} & 54.7 \\
\hline High & $3.37 \pm 10.47$ & $3.13 \pm 9.12$ & .86 & .64 & {$[.41, .80]$} & 33.4 \\
\hline Total & $402.45 \pm 103.24$ & $360.51 \pm 98.92$ & $<.001$ & .70 & {$[.40, .85]$} & 49.8 \\
\hline \multicolumn{7}{|c|}{ Leisure-time physical activity } \\
\hline Low & $24.92 \pm 36.47$ & $23.93 \pm 32.72$ & .84 & .65 & {$[.42, .80]$} & 18.4 \\
\hline Moderate & $43.36 \pm 37.78$ & $41.03 \pm 26.84$ & .57 & .71 & {$[.51, .83]$} & 25.0 \\
\hline High & $15.34 \pm 25.79$ & $13.45 \pm 19.11$ & .43 & .80 & {$[.63, .88]$} & 59.4 \\
\hline Total & $84.79 \pm 52.31$ & $78.41 \pm 43.38$ & .21 & .78 & {$[.63, .88]$} & 35.4 \\
\hline \multicolumn{7}{|c|}{ Total physical activity } \\
\hline Low & $351.44 \pm 83.01$ & $322.94 \pm 86.50$ & .04 & .51 & {$[.24, .71]$} & 38.3 \\
\hline Moderate & $114.42 \pm 71.90$ & $103.53 \pm 67.25$ & .20 & .71 & {$[.52, .84]$} & 45.2 \\
\hline High & $19.65 \pm 30.23$ & $16.24 \pm 22.84$ & .21 & .80 & {$[.66, .89]$} & 86.0 \\
\hline Total & $487.43 \pm 98.70$ & $443.99 \pm 104.95$ & $<.001$ & .70 & {$[.39, .85]$} & 42.2 \\
\hline
\end{tabular}

Note. PARA-SCI.CZ scores represent the average number of minutes of activity per day. $p=$ statistical significance for the paired samples $t$-tests comparing Week 1 and Week 2 values; $\mathrm{ICC}=$ intraclass correlation coefficient; $\mathrm{CI}=$ confidence interval; $\mathrm{CoV}=\mathrm{coef}-$ ficient of variation within subjects.

and high and total PA in LTPA. This suggested that at the group level, PARA-SCI scores were not very stable across the test-retest period. Inspection of the coefficients of variation indicated that overall, there was large within-subject variability (from $18.4 \%$ to $86 \%$ ).

\section{Discussion}

Was it relevant to determine PARA-SCI.CZ reliability and validity, when the translated semi-structured interview guide was only intended for Czech specialists physiotherapists, sports educators, nutrition therapists and, eventually, for doctors who included the interview into a battery of their professional check-ups? However, if we wanted to use the data obtained through the PARA-SCI.CZ for scientific purposes, it was desirable that the Czech version of the semi-structured interview is standardized.

It was a significant challenge to establish concurrent validity of an activity measure for people with SCI because there were limitations to using virtually every gold standard measure of activity within the SCI population. For instance, we could not use the data from heart rate monitors as a criterion because exercise intensity was not reflected in the heart rate responses of people with the sympathetic decentralization characteristic of injuries above Th6 (Cuccurullo, 2010;
Krassioukov \& West, 2014; Phillips et al., 1998). The authors of PARA-SCI used the ambulatory spirometer (Cosmed K4b2, Rome, Italy) during the validity study. The device could hardly be used to measure ADL activities that were part of the morning and evening routines (e.g., changing clothes) and would also be difficult for participants to wear for more than 5 hours a day (Martin Ginis et al., 2005). Due to the presence of the limits described above the authors of PARA-SCI demonstrated adequate criterion validity only for leisure-time PA (Latimer, Martin Ginis, \& Arbour, 2006; Martin Ginis et al., 2005).

However we decided to establish the concurrent validity, which showed a relationship between the Czech version of PARA-SCI and an accelerometer Actigraph GT3X+, even though we were aware of the fact that the motion sensors hardly detected changes in intensity due to changes in resistance (e.g., wheeling uphill), and they had difficulty detecting seated activities when there was minimal movement of the body's centre of gravity (Dishman, Washburn, \& Schoeller, 2012). A similar validity strategy had been used for example by the authors Warms and Belza (2004). The methodological approaches of the authors GarcíaMassó et al. $(2013,2015)$ and Learmonth et al. (2016) had served as a model for the compilation of methodology and data evaluation in our validity study. 
Reliability of the original PARA-SCI version was determined in a sample of 102 persons (50 persons with paraplegia and 52 persons with quadriplegia). Of the total 53 people with paraplegia, we managed to do a second phone call only in 39 paraplegic participants. Martin Ginis et al. (2005) observed higher ICC values $(.79, p=.58)$, that correspond to a good level of reliability. However, we observed lower ICC values (.70, $p$ $<.001)$ which suggests only the moderate level of testretest reliability for overall PA. Alternatively, it could be a consequence of our focus only on people with paraplegia, whose functional capacities were significantly greater compared with people with quadriplegia. It could therefore be expected that people with paraplegia had a more varied PA regime than those with a more severe disability (quadriplegia), who were reliant on help by an assistant (Bryce, 2010; Cuccurullo, 2010; Harvey, 2007).

The choice of the method for determining the reliability of PARA-SCI.CZ could be considered the limitation of the research. Most often, and it was the same in our case, we anticipated random measurement errors in terms of time fluctuations and reliability as stability over time, as measured by the test-retest method. However, this type of method could only be used with relatively stable attributes (Hendl, 2012). Therefore, the result was influenced by situations where the amount of PA changed a week later, for example due to the weather, the current health condition of the participates or his/her children, the current state of mind (motivation to PA), etc.

\section{Conclusions}

Protocol PARA-SCI.CZ focusing on the subjective perception of the amount and intensity of habitual PA of persons with spinal lesions in chronic phase was translated and adapted to the Czech language. It has good content validity. The results of criterial validity and tests-retest reliability showed moderate statistical significance. The magnitude of a spinal lesion did not significantly affect the assessment of criterial validity.

A shortened cross-cultural adapted version with an instructional video has been published and distributed into rehabilitation and social centres for persons with SCI in the Czech Republic. PARA-SCI.CZ could be used in practice by physiotherapists, nutrition therapists and sports educators from the field of adapted PA. Finally, PARA-SCI.CZ could be used in the upcoming researches dealing with epidemiologic data associated with the health status and the degree of an active lifestyle of people with SCI.

\section{Acknowledgments}

This study was supported by the Internal Grant Agency of Palacký University Olomouc (grant number IGA_FTK_2017_006).

\section{Conflict of interest}

There were no conflicts of interest.

\section{References}

Actigraph. (2016). What does the "Worn on Wrist" do in the Data Scoring tab? Retrieved from https://www.actigraph. nl/en/product/11/actilife-6.html\#faq_item_49

Anneken, V., Hanssen-Doose, A., Hirschfeld, S., Scheuer, T., \& Thietje, R. (2010). Influence of physical exercise on quality of life in individuals with spinal cord injury. Spinal Cord, 48, 393-399.

Beaton, D. E., Bombardier, C., Guillemin, F., \& Ferraz, M. B. (2000). Guidelines for the process of cross-cultural adaptation of self-report measures. Spine, 25, 3186-3191.

Bryce, T. N. (2010). Spinal cord injury. New York, NY: Demos Medical.

Buchholz, A. C., McGillivray, C. F., \& Pencharz, P. B. (2003). Physical activity levels are low in free-living adults with chronic paraplegia. Obesity Research, 11, 563-570.

Carpenter, C., Forwell, S. J., Jongbloed, L. E., \& Backman, C. L. (2007). Community participation after spinal cord injury. Archives of Physical Medicine and Rehabilitation, 88, 427-433.

Cuccurullo, S. J. (2010). Physical Medicine and Rehabilitation Board review. New York, NY: Demos Medical.

Dishman, R. K., Washburn, R. A., \& Schoeller, D. A. (2012). Measurement of physical activity. Quest, 53, 295-309.

Filáková, K., Štěpánová, J., \& Jakubec, L. (2019). Posouzení vlivu dominance horní končetiny na hodnocení objemu pohybové aktivity osob s paraplegií pomocí akcelerometru actigraph GT3X+ [Assessment of the influence of upper limb dominance on the evaluation of the volume of physical activity of persons with paraplegia using the ActiGraph GT3X+ accelerometer]. Tělesná kultura, 41, 56-63.

García-Massó, X., Serra-Añó, P., Garciá-Raffi, L. M., SánchezPérez, E. A., Lopéz-Pascual, J., \& Gonzalez, L. M. (2013). Validation of the use of Actigraph GT3X accelerometers to estimate energy expenditure in full time manual wheelchair users with spinal cord injury. Spinal Cord, 51, 898-903.

García-Massó, X., Serra-Añó, P., Gonzalez, L. M., Ye-Lin, Y., Prats-Boluda, G., \& Garcia-Casado, J. (2015). Identifying physical activity type in manual wheelchair users with spinal cord injury by means of accelerometers. Spinal Cord, 53, 772-777.

Garshick, E., Kelley, A., Cohen, S. A., Garrison, A., Tun, C. G., Gagnon, D., \& Brown, R. (2005). A prospective assessment of mortality in chronic spinal cord injury. Spinal Cord, 43, 408-416.

Ginis, K. A., Latimer, A. E., McKechnie, D., McCartney, N., Hicks, A. L., Bugaresti, J., \& Craven, C. B. (2003). Using 
exercise to enhance subjective well-being among people with spinal cord injury: The mediating influences of stress and pain. Rehabilitation Psychology, 48, 157-164.

Harvey, L. A. (2007). Management of spinal cord injuries: A guide for physioterapists. London, United Kingdom: Churchill Livingstone.

Hemphill, J. F. (2003). Interpreting the magnitude of correlation coefficients. American Psychologist, 58, 78-87.

Hendl, J. (2012). Přehled statistických metod [Overview of statistical methods]. Prague, Czech Republic: Portál.

Hetz, S. P., Latimer, A. E., Buchholz, A. C., \& Martin Ginis, K. A. (2009). Increased participation in activities of daily living is associated with lower cholesterol levels in people with spinal cord injury. Archives of Physical Medicine and Rehabilitation, 90, 1755-1759.

Hetz, S. P., Latimer, A. E., \& Martin Ginis, K. A. (2009). Activities of daily living performed by individuals with SCI: Relationships with physical fitness and leisure time physical activity. Spinal Cord, 47, 550-554.

Hicks, A. L., Martin, K. A., Ditor, S. D., Latimer, A. E., Craven, C., Bugaresti, J., \& McCartney, N. (2003). Long-term exercise training in persons with spinal cord injury: Effects on strength, arm ergometry performance and psychological well-being. Spinal Cord, 41, 34-43.

Koo, T. K., \& Li, M. Y. (2016). A guideline of selecting and reporting intraclass correlation coefficients for reliability research. Journal of Chiropractic Medicine, 15, 155-163.

Krassioukov, A., \& West, C. (2014). The role of autonomic function on sport performance in athletes with spinal cord injury. Paralympic Sports Medicine and Science, 6(Suppl. 8), S58-S65.

Kriz, J., Kulakovska, M., Davidova, H., Silova, M., \& Kobesova, A. (2017). Incidence of acute spinal cord injury in the Czech Republic: A prospective epidemiological study 2006-2015. Spinal Cord, 55, 870-874.

Latimer, A. E., Martin Ginis, K. A., \& Arbour, K. P. (2006). The efficacy of an implementation intention intervention for promoting physical activity among individuals with spinal cord injury: A randomized controlled trial. Rehabilitation Psychology, 51, 273-280.

Latimer, A. E., Martin Ginis, K. A., Craven, B. C., \& Hicks, A. L. (2006). The physical activity recall assessment for people with spinal cord injury: Validity. Medicine \& Science in Sports \& Exercise, 38, 208-216.
Lawshe, C. H. (1975). A quantitative approach to content validity. Personnel Psychology, 28, 563-575.

Learmonth, Y. C., Kinnett-Hopkins, D., Rice, I. M., Dysterheft, J. L., \& Motl, R. W. (2016). Accelerometer output and its association with energy expenditure during manual wheelchair propulsion. Spinal Cord, 54, 110-114.

Martin Ginis, K. A., \& Latimer, A. E. (2008). Physical Activity Recall Assessment for People with Spinal Cord Injury: Administration and scoring manual. Hamilton, ON: McMaster University.

Martin Ginis, K. A., Latimer, A. E., Hicks, A. L., \& Craven, B. C. (2005). Development and evaluation of an activity measure for people with spinal cord injury. Medicine \& Science in Sports \& Exercise, 37, 1099-1111.

Phillips, T. W., Kiratli, J. B., Sarkarati, M., Weraarchakul, G., Myers, J., Franklin, B. A., ... Froelicher, V. (1998). Effect of spinal cord injury on the heart and cardiovascular fitness. Current Problems in Cardiology, 23, 649-704.

Pokuta, J., Slavíková, S., \& Honzátková, L. (2014). Vliv nadváhy na kvalitu života lidí po poranění míchy [The influence of being overweight on the quality of life of persons with spinal injuries]. Aplikované pohybové aktivity v teorii a praxi, $5(2), 53$.

Štěpánová J., Kudláček, M., \& Bednaříková, M. (2016). Metody analýzy pohybové aktivity osob s transverzální míšní lézí: přehledová studie [Methods of analysis of physical activity among persons with spinal cord injury: A review]. Tělesná kultura, 39, 34-41.

Streiner, D. L., \& Norman, G. R. (2008). Health measurement scales: A practical guide to their development and use (4th ed.). Oxford, United Kingdom: Oxford University Press.

Tawashy, A., Eng, J. J., Lin, K. H., Tang, P. F., \& Hung, C. (2009). Physical activity is related to lower levels of pain, fatigue, and depression in individuals with spinal cord injury: A correlational study. Spinal Cord, 47, 301-306.

Warms, C. A., \& Belza, B. L. (2004). Actigraphy as a measure of physical activity for wheelchair users with spinal cord injury. Nursing Research, 53, 136-143.

Warms, C. A., Whitney, J. D., \& Belza, B. (2008). Measurement and description of physical activity in adult manual wheelchair users. Disability and Health Journal, 1, 236-244.

Zäch, G. A., \& Koch, H. G. (2006). Paraplegie [Paraplegia]. Basel, Germany: Karger. 